\title{
Ein neues, unkompliziertes Verfahren zur Bestimmung der Zusammensetzung binärer Flüssigkeitsgemische
}

\author{
Heinz Langhals \\ Chem. Laboratorium der Univ. Freiburg, Albertstr. 21, D-7800 Freiburg i. Br.
}

\section{A New, Uncomplicated Procedure for the Determination of the Composition of Binary Liquid Mixtures}

Summary. A new procedure for the determination of the composition of binary liquid mixtures by the use of solvatochromic dyes is described. The analysis, which consists of a simple UV/VIS absorption measurement in combination with a two parameter equation, provides an exact and rapid test.

Zusammenfassung. Ein neues Verfahren zur Bestimmung der Zusammensetzung binärer Flüssigkeitsgemische mit Hilfe solvatochromer Farbstoffe wird beschrieben. Die Analyse erfolgt durch einfache UV/VISAbsorptionsmessung und ist unter Verwendung einer Zwei-Parameter-Gleichung ein exakter Schnelltest.

Key words: Analyse von binären Flüssigkeitsgemischen, Best. von Wasser in Organ. Lösungsmitteln; Spektralphotometrie; solvatochrome Farbstoffe

\section{Einleitung}

In vorangehenden Arbeiten $[8,9]$ ist ein einfaches Verfahren beschrieben worden, mit dem man quantitativ (auch in Spuren) den Wassergehalt organischer Lösungsmittel bestimmen kann. Die Analysenmethode beruht auf einer Polaritätsmessung der zu untersuchen- $\underline{=}$

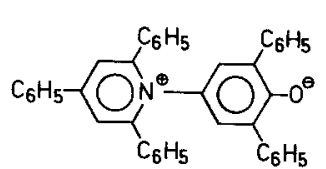<smiles>CC=CN(C)C</smiles>

$\underline{\underline{3}}$<smiles></smiles>

\section{$\stackrel{2}{=}$}

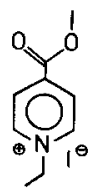

$\underline{\underline{4}}$ den Probe mit Hilfe des solvatochromen Farbstoffs 1. Die Polarität einer organischen Flüssigkeit als Funktion ihres molaren Gehaltes an Wasser $\left(c_{\mathrm{p}}\right)$ wird von der Zwei-Parameter-Formel (1) beschrieben [7], worin $c^{*}$

$E_{\mathrm{T}}=E_{\mathrm{D}} \cdot \ln \left(\frac{c_{\mathrm{p}}}{c^{*}}+1\right)+E_{\mathrm{T}}^{0}$

und $E_{\mathbf{D}}$ die empirisch zu bestimmenden Parameter der Gleichung sind. $E_{\mathrm{T}}$ ist die molare Anregungsenergie des verwendeten Farbstoffs [für 1 gilt $E_{\mathrm{T}}=E_{\mathrm{T}}(30)$ ] in der $\mathrm{zu}$ untersuchenden Probe und $E_{\mathrm{T}}^{0}$ ist der $E_{\mathrm{T}}$-Wert des reinen, wasserfreien Lösungsmittels. Die $E_{\mathrm{T}}$-Werte werden aus $\lambda_{\max }$, der Absorptionswellenlänge der Solvatochromiebande in der Probe, nach Formel (2) berechnet [13].

$E_{\mathrm{T}}=28590\left[\mathrm{kcal} \cdot \mathrm{nm} \cdot \mathrm{mol}^{-1}\right] \cdot \lambda_{\max }^{-1}$.

Wie in vorangegangenen Arbeiten $[7,10,11]$ gezeigt werden konnte, ist die Gültigkeit von Gl. (1) nicht auf Mischungen mit Wasser beschränkt. Sie beschreibt ebenfalls das Verhalten binärer Gemische organischer Lösungsmittel. $c_{\mathrm{p}}$ ist hierbei jeweils die Konzentration der polareren Komponente (das ist die Komponente, die nach der Polaritätsskala von Dimroth $u$. Reichardt $[3,13]$ den gröBeren $E_{\mathrm{T}}(30)$-Wert besitzt). Dies gestattet, das beschriebene Analysenverfahren generell bei binären Flüssigkeitsgemischen anzuwenden.

\section{Ergebnisse}

Die Gültigkeit von Gl.(1) auch für nicht wäßrige Gemische läßt sich leicht zeigen, indem man die $E_{\mathrm{T}^{-}}$ Werte von Gl. (2) gegen $\ln \left(c_{\mathrm{p}} / c^{*}+1\right)$ über mehrere Zehnerpotenzen an $c_{\mathrm{p}}$ aufträgt. In Abb. 1 ist dieser Zusammenhang für das typische Gemisch Methanol/ Dioxan dargestellt. 


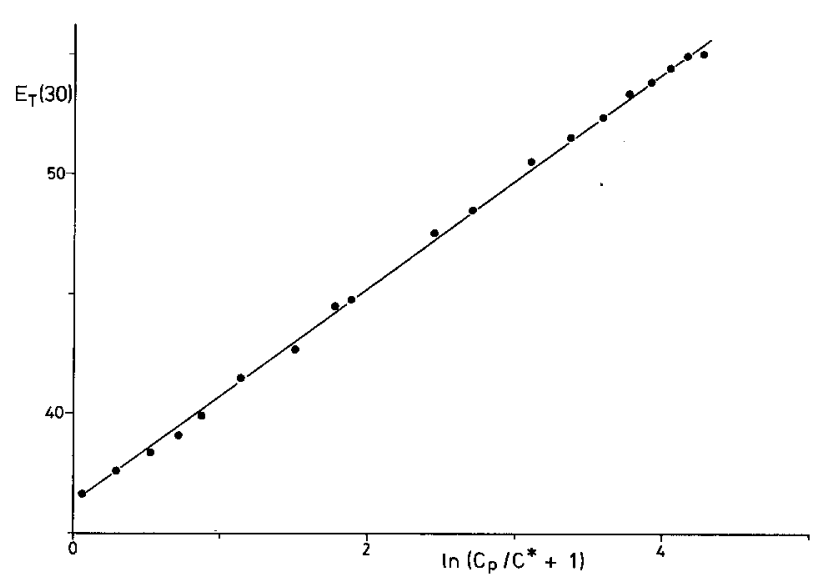

Abb. 1. $E_{\mathrm{T}}(30)$ als Funktion von $\ln \left(c_{\mathrm{p}} / c^{*}+1\right)$ für das Gemisch Methanol/Dioxan (Farbstoff 1)

Abweichungen von der erhaltenen Geraden sind rein statistischer Natur und liegen außerdem im Bereich der experimentellen Unsicherheiten bei der Bestimmung von $\lambda_{\max }$ bzw. bei der Einstellung der Eichlösungen. Die Breite der Anwendbarkeit von Gl. (1) ist aus Tabelle 1 zu ersehen, in der die $E_{\mathrm{D}}$ - und $c^{*}$-Werte sowie die Korrelationskoeffizienten für eine Reihe häufig verwendeter binärer Gemische zusammengestellt sind. Für weitere Lösungsmittelgemische können die Parameter mit Hilfe einer Eichmessung leicht bestimmt werden. Bemerkenswert ist, daß Gl. (1) nicht einmal auf Gemische zweier Flüssigkeiten beschränkt ist, wie z. B. der Fall Dodecanol/Pyridin zeigt. Die Mischung selbst muß lediglich im Meßbereich flüssig sein.

Durch Einsetzen von Gl. (2) in Gl. (1) und Umformen erhält man Gl. (3)

$c_{\mathrm{p}}=c^{*} \exp \left(\left(\frac{28590}{\lambda_{\max }}-E_{\mathrm{T}}^{0}\right) \cdot \frac{1}{E_{\mathrm{D}}}\right)-c^{*}$

mit der die Zusammensetzung des binären Gemisches berechnet wird.

Zur Bestimmung der Zusammensetzung wird wie folgt verfahren: wenig 1 wird in der Probe gelöst und $\lambda_{\max }$, das Absorptionsmaximum seiner Solvatochromiebande, bestimmt. Mit Hilfe von Gl. (3) und den Werten $E_{\mathrm{D}}, c^{*}$ und $E_{\mathrm{T}}^{0}$ von Tabelle 1 wird $c_{\mathrm{p}}$, die Konzentration der polareren Komponente, berechnet. Die Bestimmung von $c_{\mathrm{p}}$ kann damit auf eine einfache UV/VIS-Absorptionsmessung zurückgeführt werden. Eine überschlägige Gehaltsbestimmung ist sogar ohne Spektrometer, rein visuell, über einen Vergleich mit einer Farbskala [5] möglich. Für die praktische Ausführung der $c_{\mathrm{p}}$-Bestimmung ist der Farbstoff 1 besonders geeignet, da er in einem breiten Spektrum von Lösungsmitteln löslich ist und eine der stärksten bekannten Solvatochromien [13,14] aufweist, die sich zudem über den sichtbaren Bereich erstreckt. Er ist jedoch z.Zt. leider nur auf einem vielstufigen Syntheseweg zugänglich. Für Routine-Untersuchungen von binären Gemischen wäre es daher von Interesse, wenn sie mit leichter zugänglichen solvatochromen Farbstoffen ausgeführt werden könnten. Wie an anderer Stelle berichtet worden ist [11], beschreibt Gl. (1) das polare Verhalten binärer Gemische nach allen bisher untersuchten Polaritätsskalen. Deshalb sind auch die solvatochromen Farbstoffe 1-Methyl-4-(oxocyclohexadienyliden) ethyliden-1,4-dihydropyridin (MOED, 2) [2, 12], 4-Diethylamino-nitrobenzol $\left(\pi_{1}^{*}, 3\right)[4]$ und N-Ethyl-4carbomethoxypyridiniumiodid $(Z, 4)$ [6] für die Analyse binärer Gemische geeignet. Sie weisen den Vorteil auf, daß sie synthetisch wesentlich leichter zugänglich sind als $\mathbf{1}$. Farbstoff 2 absorbiert in den gebräuchlichen Lösungsmitteln hinreichend langwellig, so daß die Bestimmung von $c_{\mathrm{p}}$ auch hier visuell mit Hilfe einer Farbskala möglich ist. Bei den Farbstoffen 3 und $\mathbf{4}$ wird für die Bestimmung von $\lambda_{\max }$ ein Spektrometer benötigt. Die $E_{\mathrm{D}^{-}}$und $c^{*}$-Werte, die unter Verwendung von 2, 3 und 4 in verschiedenen binären Gemischen erhalten werden, sind ebenfalls in Tabelle 1 angegeben.

\section{Diskussion der Ergebnisse}

Die Messung kann leicht und schnell ausgeführt werden, da eine bestimmte Konzentration an Farbstoff nicht genau eingehalten werden muß. Es wird lediglich $\lambda_{\max }$ bestimmt und nicht die Extinktion der Lösung. Die Bestimmung von $\lambda_{\max }$ kann rein graphisch, ohne weitere Hilfsmittel nach der Mathiasschen Regel [1] erfolgen. Nach allen bisherigen Ergebnissen beschreibt Gl. (1) die lösungsmittelabhängige Lage von $\lambda_{\max }$ mit hoher Präzision. Eine Fehlerbetrachtung läßt sich daher auf die Untersuchung des Einflusses von Meßunsicherheiten beschränken, die nach dem Gaußschen Fehlerfortpflanzungsgesetz [15] abgeschätzt werden. (Fehler werden im folgenden mit einem $\Delta$ gekennzeichnet.) Danach setzt sich der Gesamtfehler $\left(\Delta c_{\mathrm{p}}\right)$ in $c_{\mathrm{p}}$ nach Gl. (4) aus den Einzelbeiträgen der Größen in Gl. (3) zusammen

$\Delta c_{\mathrm{p}}=\Delta c_{\mathrm{p}}(\lambda)+\Delta c_{\mathrm{p}}\left(\lambda^{0}\right)+\Delta c_{\mathrm{p}}\left(c^{*}\right)+\Delta c_{\mathrm{p}}\left(E_{\mathrm{D}}\right)$

( $E_{\mathrm{T}}^{0}$ kann nach Gl. (2) durch $\lambda^{0}$ ersetzt werden.) Die Einzelterme in Gl. (4) werden von den Gln. (5) bis (7) beschrieben. Die Parameter

$$
\begin{aligned}
& \Delta c_{\mathrm{p}}(\lambda)=\frac{28590}{E_{\mathrm{D}} \cdot \lambda^{2}}\left(c_{\mathrm{p}}+c^{*}\right) \cdot \Delta \lambda \\
& \Delta c_{\mathrm{p}}\left(c^{*}\right)=\frac{c_{\mathrm{p}}}{E_{\mathrm{D}}} \cdot \Delta c^{*} \\
& \Delta c_{\mathrm{p}}\left(E_{\mathrm{D}}\right)=\frac{c_{\mathrm{p}}+c^{*}}{28590} \cdot\left(\frac{1}{\lambda}-\frac{1}{\lambda^{0}}\right) \cdot \Delta E_{\mathbf{D}}
\end{aligned}
$$


Tabelle 1. $E_{\mathrm{D}}$ und $c^{*}$ nach Formel (1) für verschiedene Lösungsmittelgemische

\begin{tabular}{|c|c|c|c|c|c|c|c|c|c|c|}
\hline Nr. & Komponenten $^{\mathrm{a}}$ & $c_{\mathrm{p}}^{b, c}$ & $\begin{array}{l}\text { Farb- } \\
\text { stoff }\end{array}$ & $E_{\mathrm{T}}^{0 \mathrm{~d}, \mathrm{e}}$ & $c^{* \varepsilon, f}$ & $E_{\mathrm{D}}^{\mathrm{d}, \mathrm{f}}$ & $\sigma_{E_{\mathrm{D}}}^{\mathrm{g}}$ & $r^{h}$ & $n^{\mathrm{i}}$ & $c_{\mathrm{k}}^{\mathrm{c}, \mathrm{f}}$ \\
\hline 1 & 1-Butanol-Aceton & $0,01-10,9$ & 1 & 42,2 & 0,14 & 1.99 & 0,013 & 0,99952 & 31 & - \\
\hline 2 & Ethanol-Aceton & $0,02-17,1$ & 1 & 42,2 & 0,14 & 2,27 & 0,019 & 0,99939 & 28 & - \\
\hline 3 & Methanol-Aceton & $0,03-24,7$ & 1 & 42,5 & 0,10 & 2,53 & 0,019 & 0,99973 & 29 & - \\
\hline 4 & Wasser-Aceton & $0,06-55,4$ & 1 & 42,2 & 0,31 & 2,83 & 0,022 & 0,99963 & 29 & $16,5^{\mathrm{k}}$ \\
\hline 5 & N-tert.-Butylformamid-Aceton & $0,01-9,0$ & 1 & 46,0 & 0,27 & 1,87 & 0,013 & 0,99948 & 31 & - \\
\hline 6 & Ethanol-Acetonitril & $0,01-17,1$ & 1 & 46,0 & 0,10 & 1,83 & 0,012 & 0,99972 & 30 & 12,0 \\
\hline 7 & 1-Hexanol-Acetonitril & $0,01-8,0$ & 1 & 46,0 & 0,08 & 1,08 & 0,019 & 0,99876 & 29 & - \\
\hline 8 & Methanol-Acetonitril & $0,03-24,7$ & 1 & 46,0 & 0,06 & 1,83 & 0,033 & 0,99877 & 31 & - \\
\hline 9 & Wasser-Acetonitril & $0,06-49,8$ & $\hat{1}$ & 46,0 & 0,15 & 2,07 & 0,024 & 0,99877 & 27 & - \\
\hline 10 & N-tert.-Butylformamid-Benzol & $0,01-9,0$ & 1 & 34,5 & 0,01 & 2,27 & 0,031 & 0,99851 & 31 & - \\
\hline 11 & Wasser-tert.-Butylalkohol & $0,06-33,2$ & 1 & 43,9 & 1,01 & 2,82 & 0,050 & 0,99666 & 26 & - \\
\hline 12 & Wasser-tert.-Butylhydroperoxid & $0,4-7,4$ & 1 & 49,7 & 0,312 & 1,40 & 0,021 & 0,99922 & 12 & - \\
\hline 13 & 1-Butanol-Dimethylformamid & $0,01-10,9$ & $\mathbf{1}$ & 43,8 & 1,64 & 3,20 & 0,02 & 0,99936 & 30 & 8,7 \\
\hline 14 & Ethanol-Dimethylformamid & $0,02-17,1$ & 1 & 43,8 & 0,607 & 2,78 & 0,04 & 0,99792 & 30 & 13,7 \\
\hline 15 & Methanol-Dimethylformamid & $0,03-24,7$ & 1 & 43,8 & 0,696 & 3,55 & 0,05 & 0,99745 & 31 & - \\
\hline 16 & Wasser-Dimethylformamid & $0,06-38,8$ & 1 & 43,8 & 11,43 & 9,24 & 0,18 & 0,99527 & 27 & - \\
\hline 17 & 1-Butanol-Dimethylsulfoxid & $0,01-10,9$ & 1 & 45,0 & 4,29 & 4,93 & 0,1 & 0,99475 & 30 & 7,7 \\
\hline 18 & Ethanol-Dimethylsulfoxid & $0,02-17,1$ & 1 & 45,0 & 2,69 & 3,95 & 0,05 & 0,99794 & 30 & 13,7 \\
\hline 19 & Acetonitril-1,4-Dioxan & $0,02-19,1$ & 1 & 36,0 & 0,77 & 3,23 & 0,031 & 0,99909 & 29 & - \\
\hline 20 & 1-Butanol-1,4-Dioxan & $0,01-10,1$ & 1 & 36,0 & 0,90 & 5,39 & 0,026 & 0,99973 & 30 & - \\
\hline 21 & Ethanol-1,4-Dioxan & $0,02-17,1$ & 1 & 36,0 & 0,72 & 4,99 & 0,030 & 0,99975 & 30 & - \\
\hline 22 & Methanol-1,4-Dioxan & $0,03-24,7$ & 1 & 36,0 & 0,35 & 4,55 & 0,037 & 0,99922 & 30 & - \\
\hline 23 & Nitromethan-1,4-Dioxan & $0,02-18,6$ & 1 & 36,0 & 1,01 & 3,49 & 0,026 & 0,99929 & 31 & - \\
\hline 24 & Propionitril-1,4-Dioxan & $0,01-14,1$ & 1 & 36,0 & 1,41 & 3,33 & 0,038 & 0,99834 & 30 & - \\
\hline 25 & Wasser-1,4-Dioxan & $0,6-55,4$ & 1 & 36,0 & 0,58 & 4,34 & 0,054 & 0,99922 & 22 & $22,0^{1}$ \\
\hline 26 & 1-Butanol-Nitromethan & $0,01-10,9$ & 1 & 46,3 & 0,06 & 1,43 & 0,015 & 0,99914 & 31 & 6,5 \\
\hline 27 & Ethanol-Nitromethan & $0,02-17,1$ & 1 & 46,3 & 0,03 & 1,41 . & 0,030 & 0,99704 & 30 & 8,5 \\
\hline 28 & Methanol-Nitromethan & $0,03-22,2$ & $\hat{1}$ & 46,3 & 0,01 & 1,66 & 0,018 & 0,99947 & 29 & 10,0 \\
\hline 29 & Aceton-Pyridin & $0,03-12,2$ & 1 & 40.2 & 32,06 & 4,01 & 0,13 & 0,98710 & 28 & - \\
\hline 30 & 1-Dodecanol-Pyridin & $0,004-4,5$ & 1 & 40,2 & 0,89 & 2,90 & 0,03 & 0,99901 & 30 & 3,0 \\
\hline 31 & Ethanol-Pyridin & $0,02-17,1$ & 1 & 40,2 & 12,75 & 9,64 & 0,17 & 0,99554 & 31 & - \\
\hline 32 & 1-Hexanol-Pyridin & $0,01-8,0$ & 1 & 40,2 & 1,11 & 2,90 & 0,041 & 0,99763 & 30 & - \\
\hline 33 & Methanol-Pyridin & $0,03-24,7$ & 1 & 40,2 & 5,84 & 6,92 & 0,081 & 0,99802 & 31 & - \\
\hline 34 & Nitromethan-Pyridin & $0,02-18,6$ & 1 & 40,2 & 13,62 & 6,46 & 0,14 & 0,99384 & 30 & - \\
\hline 35 & tert.-Pentylalkohol-Pyridin & $0,005-8,3$ & 1 & 40,2 & 0,95 & 1,02 & 0,025 & 0,99341 & 29 & - \\
\hline 36 & Wasser-Pyridin & $0,06-49,8$ & 1 & 40,2 & 5,48 & 7,09 & 0,12 & 0,99599 & 29 & - \\
\hline 37 & 1-Butanol- $-\mathrm{CS}_{2}$ & $0,01-10,9$ & 1 & 32,6 & 0,03 & 2,42 & 0,028 & 0,99906 & 30 & 7,5 \\
\hline 38 & 1-Octanol- $\mathrm{CS}_{2}$ & $0,01-6.4$ & 1 & 32,6 & 0,06 & 2.83 & 0,055 & 0,99676 & 30 & 4,0 \\
\hline 39 & Pinakolon- $\mathrm{CS}_{2}$ & $0,3-8,0$ & 1 & 32,6 & 7,89 & 9,30 & 0,26 & 0,99459 & 16 & - \\
\hline 40 & 1-Butanol-Tetramethylharnstoff & $0,01-10,9$ & 1 & 41,0 & 0,91 & 3,56 & 0,03 & 0,99936 & 31 & - \\
\hline 41 & Ethanol-Tetramethylharnstoff & $0,02-17,1$ & 1 & 41,0 & 0,81 & 3,51 & 0,03 & 0,99925 & 31 & - \\
\hline 42 & Methanol-Aceton & $0,03-24,7$ & 2 & 49,3 & 0,31 & 2,25 & 0,01 & 0,99952 & 30 & - \\
\hline 43 & Wasser-Ethanol & $0,06-55,4$ & 2 & 55,9 & 5,47 & 2,04 & 0,06 & 0,99168 & 24 & 30 \\
\hline 44 & Wasser-Ethanol & $0,06-55,4$ & 3 & 72.9 & 3,08 & $-1,71$ & 0,02 & $-0,99846$ & 31 & 30 \\
\hline 45 & Ethanol-n-Heptan & $0,02-17,1$ & 3 & 78,7 & 3,05 & $-3,19$ & 0,04 & $-0,99768$ & 31 & - \\
\hline 46 & Ethanol-Acetonitril & $0,02-17,1$ & 4 & 72,1 & 0,48 & 2,15 & 0,02 & 0,99866 & 47 & 6,8 \\
\hline 47 & Methanol-Aceton & $0,03-24,7$ & 4 & 66,3 & 0,65 & 4,66 & 0,014 & 0,99993 & 31 & - \\
\hline 48 & Methanol-1,4-Dioxan & $0,03-24,7$ & 4 & 63,7 & 2,66 & 8,52 & 0,18 & 0,99384 & 31 & - \\
\hline
\end{tabular}

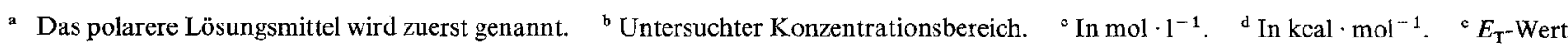
der unpolareren Komponente (s. a. Lit. [13]). ${ }^{\mathrm{f}}$ Siehe Text. $\quad{ }^{\mathrm{g}}$ Varianz von $E_{\mathrm{D}} \cdot{ }^{\mathrm{h}}$ Korrelationskoeffizient bei Anwendung von Gl. (1). ${ }^{\mathrm{i}}$ Anzahl der Meßpunkte. ${ }^{\mathrm{k}}$ Für $c_{\mathrm{p}}>c_{\mathrm{k}}$ gilt $E_{\mathrm{D}}=6,8$ und $c^{*}=6,1 .{ }^{1}$ Für $c_{\mathrm{p}}>c_{\mathrm{k}}$ gilt $E_{\mathrm{D}}=20,0$ und $c^{*}=19,1$

von Gl. (3), $E_{\mathrm{D}}$ und $c^{*}$, werden als Mittelwerte über eine Meßreihe erhalten, und deshalb sollte der Beitrag ihres Fehlers zu $\Delta c_{\mathrm{p}}$ verhältnismäßig gering sein. Gleiches gilt für $c_{\mathrm{p}}\left(\lambda^{0}\right)$, dessen Berechnung analog Gl. (5) erfolgt. Den Hauptbeitrag zum Gesamtfehler liefert bei einer Einzelmessung daher Gl. (5), die im folgenden behan- delt wird. Nach G1. (5) ist der Fehler in $c_{\mathrm{p}}$ bei konstanter Meßunsicherheit ( $4 \lambda$ ) direkt proportional zu der Summe von $c_{\mathrm{p}}$ und $c^{*}$ und umgekehrt proportional zu $E_{\mathrm{D}}$ und $\lambda^{2}$. Für $c_{\mathrm{p}} \ll c^{*}$ ist der absolute Meßfehler in $c_{\mathrm{p}}$ konzentrationsunabhängig, während er für $c_{\mathrm{p}} \gg c^{*}$ linear abhängig von $c_{\mathrm{p}}$ wird. In diesem Falle ist der 
relative Meßfehler in $c_{\mathrm{p}}$ konzentrationsunabhängig. Dies ist für die Praxis besonders wichtig, da der relative Fehler innerhalb des Meßbereichs konstant sein sollte. Ist $c^{*}$ klein, kann dies sogar über mehrere Zehnerpotenzen an $c_{\mathrm{p}}$ erfüllt sein.

Die umgekehrte Proportionalität zwischen $c_{\mathrm{p}}(\lambda)$ und $\lambda^{2}$ hat für die praktische Auswahl von solvatochromen Farbstoffen Konsequenzen. Zur Erzielung eines minimalen Fehlers in $c_{\mathrm{p}}$ sollte ein solvatochromer Farbstoff mit möglichst langwelliger Absorption verwendet werden (z. B. 1 oder 2). Darüberhinaus sind in wenig polaren Lösungen Farbstoffe mit stark negativer Solvatochromie $\left(E_{\mathrm{D}}>0\right)$ vorzuziehen (möglichst langwellige Absorption), während in stark polaren Lösungen solche mit positiver Solvatochromie $\left(E_{\mathbf{D}}<0\right)$ günstiger sind.

Für eine genaue Messung von $c_{\mathrm{p}}$ sollten sich die Polaritäten der Komponenten im binären Gemisch hinreichend unterscheiden. Einige Gemische (s. Tabelle 1) weisen Besonderheiten auf: bei hohen Konzentrationen an polarerer Komponente $\left(c_{\mathrm{p}}>5 \mathrm{~mol} \cdot \mathrm{1}^{-1}\right)$ geht die Funktion (1) abrupt in eine andere, analoge mit anderen $E_{\mathrm{D}^{-}}$und $c^{*}$-Werten über. Dies stellt jedoch keine Einschränkung des Analysenverfahrens dar.

\section{Experimenteller Teil}

Die UV/VIS-Spektren wurden mit dem UV-Spektrometer DMR-21 der $\mathrm{Fa}$. Zeiss aufgenommen. Für eine überschlägige Bestimmung von $\lambda_{\max }$ genügt der Farbvergleich mit einer Farbskala : Absorptionswellenlänge-Absorptionsfarbe, z. B. Lit. [5]. Die verwendeten Farbstoffe sind nach Literatur-Angaben $[2-4,11,12]$ synthetisiert worden.

Bestimmung von $c_{\mathrm{p}}$. Eine Glas- oder Quarzküvette von $1 \mathrm{~cm}$ Schichtdicke, mit einem Stopfen versehen, wird mit der Flüssigkeitsprobe gegebenenfalls unter Feuchtigkeitsausschluß gefüllt. Eine geringe Menge des solvatochromen Farbstoffs wird gelöst, bis die Extinktion im Maximum der Solvatochromiebande des Farbstoffs einen Wert zwischen 0,7 und 1,0 erreicht. $\lambda_{\max }$ wird bestimmt. Seine genaue Lokalisierung kann mit der Mathiasschen Regel [1] erfolgen. Der so gefundene Wert wird mit den entsprechenden Konstanten von Tabelle 1 in Gl. (3) eingesetzt und $c_{\mathrm{p}}$ berechnet. $c_{\mathrm{p}}$ kann dann in bekannter Weise in Gew.- \% oder Vol.- \% der polaren Komponente umgerechnet werden.

Bestimmung der Parameter $E_{\mathbf{D}}$ und $c^{*}$ für weitere binäre Gemische und solvatochrome Farbstoffe. In einem $10 \mathrm{ml}$-Meßkolben werden bei $25^{\circ} \mathrm{C} 0,5,1,2 \ldots 9 \mathrm{ml}$ polarere Komponente einpipettiert und mit der unpolaren Komponente aufgefüllt. Die Lösung wird mit einem solvatochromen Farbstoff angefärbt und wie oben $\lambda_{\max }$ bestimmt. Nach Umrechnen des Gehalts an polarerer Komponente in $c_{\mathrm{p}}$ wird in einem Diagramm $E_{\mathrm{T}}$, berechnet nach Gl. (2), gegen $\ln c_{\mathrm{p}}$ aufgetragen. Durch den linearen Teil der Kurve wird eine Gerade gelegt und ihre Steigung $\left(E_{\mathrm{D}}\right)$ und der Ordinatenabschnitt (b) bestimmt. Die Berechnung von $c^{*}$ erfolgt nach Gl. (8).

$c^{*}=\exp \left[\left(E_{\mathrm{T}}^{0}-b\right) / E_{\mathrm{D}}\right]$.

Für diese Prozedur steht das Rechenprogramm POLAR ${ }^{1}$ (in FORTRAN V) zur Verfügung, das auch die Meßwerte im nichtlinearen Teil der Kurve berücksichtigt. Die $E_{\mathrm{D}^{-}}$und $c^{*}$-Werte von Tabelle 1 sind mit diesem Rechenprogramm an der Universität Freiburg berechnet worden.

Beispiel für eine $c_{\mathrm{p}}$-Bestimmung. Der Acetongehalt einer eingestellten Dioxanlösung $\left(12,4 \mathrm{~mol} \cdot \mathrm{1}^{-1}\right)$ soll mit Hilfe von Gl. (3) bestimmt werden. Hierfür wird in einer Probe wenig 1 gelöst und $\lambda_{\max }$ bestimmt: $546 \mathrm{~nm}$. Mit Hilfe von Gl. (3) und den $E_{\mathrm{D}^{-}}$und $c^{*}$-Werten von Tabelle 1 wird der Gehalt an Aceton berechnet: $12,5 \mathrm{~mol} \cdot 1^{-1}$; Fehler 0,8\%.

Herrn Prof. Dr. C. Rüchardt und der Deutschen Forschungsgemeinschaft wird für die Unterstützung dieser Arbeit gedankt.

\section{Literatur}

1. Brdicka, R.: Grundlagen der Physikalischen Chemie, 10. Aufl., S. 252, 503. Berlin: VEB 1972

2. Brooker, L. G. S., Keyes, G. H., Heseltine, D. W. : J. Am. Chem. Soc. 73, 5350 (1951)

3. Dimroth, K., Reichardt, C., Siepmann, T., Bohlmann, F.: Liebigs Ann. Chem. 661, 1 (1963)

4. Kamlet, M. J., Hall, T. N., Boykin, J., Taft, R. W. : J. Org. Chem. 44, 2599 (1979)

5. Klessinger, M.: Chemie in unserer Zeit 12, 1 (1978)

6. Kosower, E. M.: J. Am. Chem. Soc. 80, 3253 (1958)

7. Langhals, H.: Nouv. J. Chimie 5, 97 (1981)

8. Langhals, H.: Fresenius Z. Anal. Chem. 305, 26 (1981)

9. Langhals, H.: DOS 3043897.2 vom 21. 11. 1980

10. Langhals, H.: DOS 3043984.0 vom 21. 11. 1980

11. Langhals, H.: Chem. Ber. (im Druck)

12. Minch, M. J., Shah, S. S.: J. Chem. Educ. 54, 709 (1976)

13. Reichardt, C.: Angew. Chem. 91, 119 (1979)

14. Reichardt, C.: Solvent Effects in Organic Chemistry. Weinheim: Verlag Chemie 1979

15. Zachmann, H. G.: Mathematik für Chemiker, 2. Aufl., S. $617-$ 619, Weinheim: Verlag Chemie 1974

Eingegangen am 28. Februar 1981

1 Das Programm wird auf Wunsch zur Verfügung gestellt 